\title{
Correlation between Students' Understanding on Derivative and Integral Calculus with Thermodynamics
}

\author{
Duden Saepuzaman \\ Physics Education Department \\ Universitas Pendidikan Indonesia \\ Bandung, Indonesia \\ dsaepuzaman@upi.edu
}

\author{
Aldi Zulfikar \\ Physics Education Department \\ Universitas Pendidikan Indonesia \\ Bandung, Indonesia \\ aldizulfikar@student.upi.edu
}

\author{
Denni Yulius Girsang \\ Physics Education Department \\ Universitas Pendidikan Indonesia \\ Bandung, Indonesia \\ denni.yulius.girsang@student.upi.edu
}

\begin{abstract}
This research was conducted in order to find the correlation between students' understanding on derivative and integral calculus with thermodynamics. The instrument used in this research was a test comprising of essays and interview questions. The participants in this research were 69 prospective physics teachers who studied in Physics Education Department. The result indicated that there was a correlation between students' understanding on calculus with thermodynamics. Results from interview also indicated that understanding of calculus is essential in order to understand thermodynamics. Therefore, this research suggests the needs for further improving learning process in calculus subject in order to improve other subjects that implemented calculus such as in thermodynamics. Therefore, the students could understand both the concept and the mathematical representation.
\end{abstract}

Keywords - correlation; thermodynamics; derivative; integral

\section{INTRODUCTION}

Physics is a subject that tries to understand the behavior and structure of matter [1] which could be perceived through observations of phenomena. In order to understand these phenomena thoroughly, some methods of representations are necessary. One of these methods is the use of modeling. A model representation of a certain phenomenon could be presented through verbal, computational, mathematical, diagrams, or graphs [2]. Mathematical representations, in particular, are very familiar in physics because it could be derived in order to describe other phenomena and visualize relation between involved variables [5]. Some examples of physics subjects which utilize a lot of derivatives and integrals are mechanics, electricity, and thermodynamics. In mechanics, position as a function of time can be derived in order to find velocity (first derivative) and acceleration (second derivative). In electricity, the current flowing within a wire is the first derivative of charge as a function of time. In thermodynamics, which will be the main topic in this research, the relation between each thermodynamics coordinates are evaluated through the help of partial derivatives or that the work (energy) could be obtained from the integral of pressure as the volume changes infinitesimally. It is, therefore, very likely that there is a strong relation between cognitive understanding (based on test results) of derivative and integral with particular physics subjects which utilize a lot of them, and thermodynamics is the subject that will be focused and discussed in this research.

(a)

$$
\begin{aligned}
& \text { By using the rules of implicit derivatives, } \\
& \text { determine the value of (dy/dx) of } \\
& x^{2} y^{2}+\cos \left(x y^{2}\right)+4 x y=4
\end{aligned}
$$

(b)

Fig. 1. (a) An example of Calculus question from original document. (b) Translation from the original document

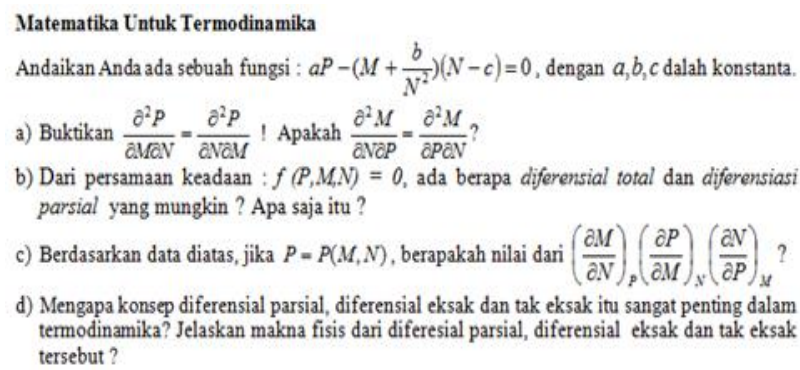
termodinamika? Jelaskan makna fisis dari diferesial parsial, diferensial eksak dan tak eksak tersebut ?

(a) 


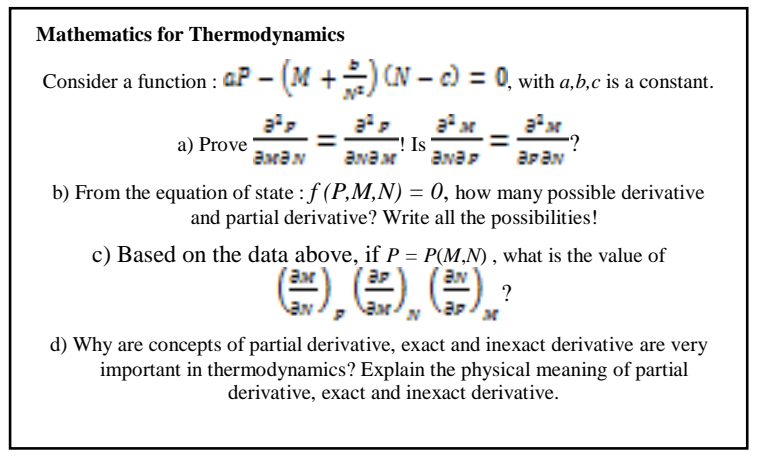

(b)

Fig. 2. (a) An example of Thermodynamics question from original document. (b) Translation from the original document

Thermodynamics can be defined as the science of energy [3], which evaluate the relation between its' thermodynamics coordinates (volume, temperature, pressure, etc.) in a closed system at the state of equilibrium [3], based on its' equation of state [4]. In order to understand the relation between these thermodynamics coordinates, comprehension of derivatives and integrals are essential because the subject utilizes a lot of mathematical representations to describe phenomena and most of the equations are manipulated those derivatives and integrals in order to find relations between particular coordinates. Derivatives and integrals deal with very small changes of certain variable. For example, if $\mathrm{A}$ is a function of $B$, written as $A=A(B)$, which means that the value of $A$ will change when $\mathrm{B}$ changes and to observe very small changes, which is close to zero (because thermodynamics coordinates changes happens very slowly), there is a need of an operational tool to observe the changes. Partial derivative, for example, is used in order to describe the very small changes of certain coordinate because of other coordinate's changes, assuming all other coordinates are at constant. Other example, is the use of Euler's formula in order to find whether or not a function is exact or not, which is necessary in order to derive it's equation of state. In order to find the work from one point to another, it is important to understand how to integrate certain values. Integrals will be used in order to find a function from its' derivative form or to find a value of certain coordinates which is a function of some other coordinates.

\section{RESEARCH METHOD}

\section{A. Design of Research}

This research was conducted in order to find the correlation between two variables, students' academic achievements in calculus and in thermodynamics. Students' academic achievements in calculus and in thermodynamics were represented by the test results which conducted separately. Afterwards, a few students are interviewed in order to find proofs that could help explain the result. In calculus, the scores were obtained from the 1st Unit Test of the subject which consisted of derivatives and integrals, while for thermodynamics the scores were obtained from the Mid-Term Test which consisted of basics of thermodynamics, specifically thermodynamics coordinates (volume, pressure, temperature, energy) and equation of state for ideal gas. The test for calculus is conducted in 2013 while thermodynamics was conducted in 2015. The reason is because students contracted calculus in the 1 st semester and contracted thermodynamics in the 4 th semester.

\section{B. Participants}

Participants of this research are prospective physics teachers who studied in Physics Education Department at Universitas Pendidikan Indonesia. The participants' age was varied from 17 to 19 years old, while that in thermodynamics was varied from 19-21 years old for the same participants. In total, there are 16 male and 53 female participants in this research.

\section{Assessment Tools}

The instrument used in this research is test questions in a form of essays, which consist of 9 questions for calculus and 5 questions for thermodynamics. An example of the question for calculus and thermodynamics are shown in Fig. 1 and Fig. 2 respectively.

\section{RESULTS AND DISCUSSION}

Students results are divided into groups based on the score of each subject. Results for each subject are divided into 3 categories, which are low (L), medium (M), and high $(\mathrm{H})$. Based on this categorization, and ordering the score in particular order, calculus' score followed with thermodynamics', the possible results are LL (low-low), LM (low-medium), LH (low-high), ML (medium-low), MM (medium-medium), $\mathrm{MH}$ (medium-high), HL (high-low), HM (high-medium), and $\mathrm{HH}$ (high-high). Based on this categorization method, it is possible to see the relation between the two scores.

The data shown in Fig. 3 had been tested in regards to its homogeneity and normality. Based on data correlation analysis of Karl Pearson, by processing the data which consist of the test score from calculus' and thermodynamics' respectively for each person, the value of correlation coefficient $r=0.56$. Based on the table provided by Sugiyono [6], the correlation is considered medium, which means the result from calculus has quite an impact on the result of thermodynamics, in other words, the result of calculus and thermodynamics is not negligible. This result is also strengthened by the fact that based on independency test, both variables are not independent.

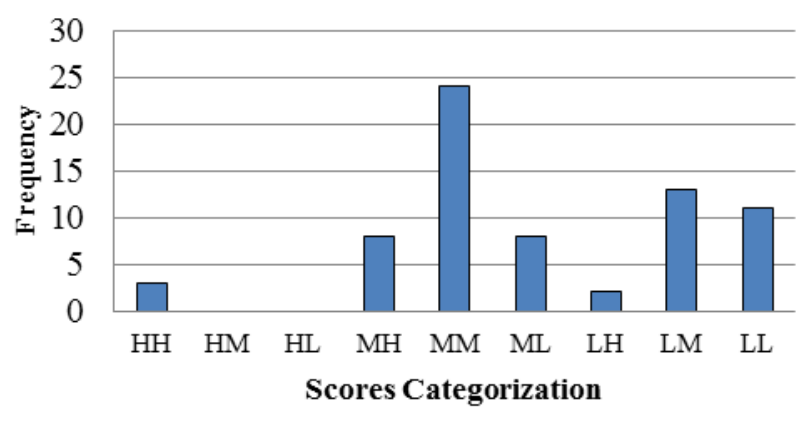

Fig. 3. Frequency of Calculus-Thermodynamics' Score 
In regards to Fig. 3, there are some important things that need to be emphasized. The first point is in regards to students with high result on calculus' test. It was shown in the graph that all students who gets high result on calculus also have high result in thermodynamics. Based on the results from interview, because students are able to understand the mathematics beforehand, the focus of learning in classroom could be directed more to the conceptual understanding which was explained very well, which provides students with a more complete understanding of how the mathematics work and the concept behind it.

The second point is about the fact that students with high achievement in calculus achieved just as high in thermodynamics and the same thing also applied for the majority of students with medium achievement. These facts indicated that there is a relation between mathematical or calculus achievement with thermodynamics achievements. A slightly different result was found in students with low achievement. There some factors related to this particular case. One of the most influential factors is the fact that students still have a year, including short semester, to study about calculus in calculus for short semester, first mathematical physics in 2nd semester and second mathematical physics in 3rd semester. It opens up to a lot of opportunity to improve students' mathematical skills. Especially for students who achieved low on calculus, but high in thermodynamics, it was because they joined the short semester class which provides them with stronger understanding of how the mathematical equations and operations work.

The third important point that needs emphasizes here is the fact some medium achiever students for calculus got lower during thermodynamics. The reason of this is because students could have forgotten about some important things about derivative and integral because of the time span between the tests. Another factor might be because students with medium achievement needs to focus on two things, regain the understanding mathematical derivation or integration and conceptual understanding necessary in order to understand thermodynamics more than just mathematical equations, but also the physical significances behind it.

\section{CONCLUSION}

Based on the research, it can be concluded that the result of thermodynamics subject was affected by students comprehension in derivatives and integrals. The correlation between the two variable represented by correlation coefficient $r$ of 0.56 . From this result, it is important to note that in order to improve the quality of achievements in thermodynamics, and other subjects which also utilize a lot of derivatives and integrals, there is a strong need for improvement in the learning process in calculus, so that students could focus on the concepts, instead of trying to figure out how the mathematics work. That way, students could perceive a more holistic understanding of the subject, and not only partially, either to understand only the general concept, or only as a collection of equations.

\section{References}

[1] D. C. Giancoli, Physics Principles with Application (6th Edition), New Jersey: Pearson Education, Inc., 2005.

[2] B. M. Zwickl and D. Hu, "Model based reasoning in the physics laboratory: Framework and initial results," American Physical Society, pp. 2-3, 2015.

[3] Y. A. Cengel and M. A. Boles, Thermodynamics: An Engineering Approach (5th Edition), New York: McGraw-Hill, 2006.

[4] M. W. Zemansky and R. H. Dittman, Heat and Thermodynamics, New York: McGraw-Hill, 1997.

[5] J. R. Thompson, B. R. Bucy and D. B. Mountcastle, "Assessing Student Understanding of Partial Derivatives in Thermodynamics," American Institute of Physics, pp. 77-80, 2005.

[6] Sugiyono, Metode Penelitian Kuantitatif Kualitatif dan R\&D, Bandung: Alfabeta, 2008. 